\title{
Lessons learned from the detection of ischemia in asymptomatic diabetics (DIAD) study
}

\author{
Frans J. Th. Wackers, MD and Lawrence H. Young, MD
}

Over 20 million Americans and 200 million people worldwide are estimated to have type 2 diabetes. Cardiovascular disease is the leading cause of death and a major cause of morbidity in diabetic patients, and therefore represents a serious global health care problem. It is well recognized that diabetic patients with symptomatic coronary artery disease are at high risk for adverse cardiac events. However, there is significant concern about cardiovascular risk in others with no known history of heart disease or symptoms of myocardial ischemia. Professional organizations have advocated aggressive primary preventative therapies with statins, aspirin, anti-hypertensives, and glycemic control. However, despite greater utilization of these therapies, patients with diabetes remain at risk for myocardial infarction and sudden cardiac death. Concern for these complications has generated enthusiasm for screening with specialized cardiac testing. The rationale for screening is that the early identification of ischemia or structural CAD would lead to additional therapies, including coronary revascularization, which might prevent myocardial infarction of sudden death.

The Detection of Ischemia in Asymptomatic Diabetics (DIAD) study (www.clinical trials.gov identifier \# NCT00769275) was designed not only to better understand the prevalence and predictors of inducible ischemia, but also to determine whether screening prevents cardiac events in asymptomatic patients with type 2 diabetes. The main outcome results of DIAD have been published recently in the Journal of the American Medical Association ${ }^{1}$ and have engendered substantial discussion because of their clinical and public health implications. In this discussion, we will focus on certain aspects of the DIAD study that are germane for

From the Department of Medicine, Section of Cardiovascular Medicine, Yale University School of Medicine, New Haven, CT.

Reprint requests: Frans J. Th. Wackers, MD, Department of Medicine, Section of Cardiovascular Medicine, Yale University School of Medicine, 333 Cedar Street Fitkin-3, New Haven, CT 06520; frans. wackers@yale.edu.

J Nucl Cardiol 2009;16:855-9.

1071-3581/\$34.00

Copyright (C) 2009 by the American Society of Nuclear Cardiology. doi:10.1007/s12350-009-9138-1 appreciating its practical implications. For details of the analyses we refer to the original publications. ${ }^{1-3}$

\section{SELECTION OF PATIENTS FOR THE DIAD STUDY}

The DIAD study was designed to explore the value of routine screening with adenosine Tc-99m Sestamibi SPECT myocardial perfusion imaging (MPI) in patients with type 2 diabetes without signs or symptoms of coronary artery disease. The characteristics of the DIAD patients are important for understanding the general applicability of the results. The participants were 5075 years old and had type 2 diabetes and no clinical symptoms of CAD. ${ }^{2}$ All potential participants were required to complete the Rose questionnaire for angina or chest discomfort and those with a positive questionnaire were excluded. Patients with $>1 \mathrm{~mm}$ ST segment depression or pathological Q-waves on resting ECG, suggesting prior ischemia or myocardial infarction, were also excluded. Furthermore, candidates with any prior stress testing within the prior 3 years were also not eligible for the study.

These entry criteria appropriately excluded patients who might be referred for cardiac testing for clinical reasons. One of the criticisms expressed regarding the DIAD study, was that the patients were a self-selected and low-risk cohort. Although patients who volunteer to participate in clinical trials tend to be more concerned about their health than the general population, DIAD had an extremely high consent rate, with $66 \%$ of all eligible patients agreeing to participate. Moreover, their diabetes was well cared for with an average HbA1C level of $7.1 \%$.

On the other hand the DIAD participants had characteristics that typically raise concern about cardiac risk. Their average duration of diabetes was 8.5 years; they were older (mean 60.7 years) and often obese (mean BMI 31). More than $60 \%$ of participants had two or more additional traditional risk factors for CAD. Notably, many had very poor functional capacity: $50 \%$ of subjects were unable to walk at a slow pace during adenosine infusion. Thirty-four percent of participants reported a completely sedentary life style.

When the DIAD study was designed, the literature suggested that as many as $50-60 \%$ of patients with diabetes might have abnormal stress MPI, based on 
retrospective database analyses of patients referred for clinical stress testing. ${ }^{4-13}$ The prospective Milan study of patients in the general diabetes population found a much lower incidence of inducible ischemia (7\%), but selected healthier patients who were required to complete a full exercise test. ${ }^{14}$ The use of adenosine pharmacologic stress assured that DIAD did not exclude diabetics who were unable to perform adequate physical exercise due to diabetic neuropathy, obesity, or peripheral arterial disease. As such, DIAD was much more inclusive and included higher-risk patients.

\section{PREVALENCE OF SILENT CAD}

The first important finding of the DIAD study was that the prevalence of abnormal stress MPI was $22 \%{ }^{2}$ The majority of the abnormalities were small with only $6 \%$ that were quantified as moderate or large. Another $6 \%$ of patients had only ischemic ECG changes during adenosine infusion. The DIAD study therefore sheds a different perspective on the prevalence of silent CAD in asymptomatic diabetics than retrospective database analysis from nuclear cardiology laboratories. The latter may paint a distorted picture of the actual prevalence because of patient referral bias. Furthermore, the concept that most patients with diabetes have asymptomatic myocardial ischemia over-states the prevalence and their risk.

\section{LACK OF PROGRESSION OF ISCHEMIA}

CAD is assumed to be rapidly progressive in patients with diabetes. One of the surprises of the DIAD study was that there was no evidence for more inducible ischemia in screened patients when MPI was repeated after 3 years. Rather than a greater prevalence of abnormal MPI, there was significantly less inducible ischemia at repeat imaging: $12 \%$ vs $20 \% .^{3}$ Although a small number of patients with intervening cardiac events or coronary revascularization were excluded from this analysis, a remarkable $79 \%$ of participants with initially abnormal stress MPI at recruitment, had resolution of ischemia. This improvement was not restricted to small perfusion defects, but rather occurred regardless of the initial magnitude of abnormal MPI abnormality (Figure 1). Conversely, only $10 \%$ of participants with initially normal screening MPI developed new inducible ischemia after 3 years.

This observed resolution of asymptomatic ischemia was unanticipated, since patients with CABG or PCI were specifically excluded from participation in the repeat imaging analysis. An alternative explanation became apparent after review of the medical regimens of the participants. In the course of 3 years, there was a significant increase in the treatment of DIAD subjects

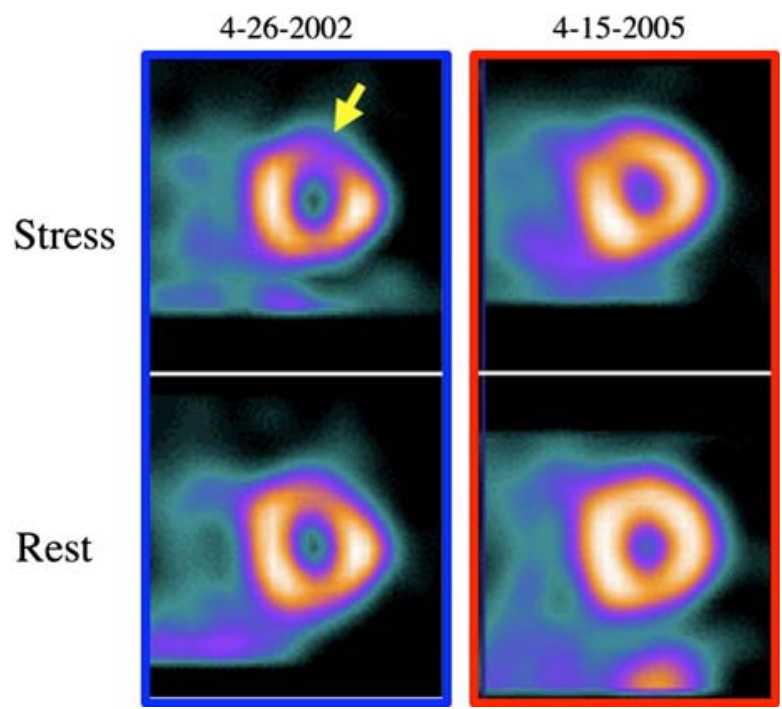

Figure 1. Representative example of a patient in the DIAD study who had resolution of inducible myocardial ischemia after 3 years. The study on April 26, 2002 showed a small but definite reversible anterior wall myocardial perfusion defect. Three years later on April 15, 2005 there is no longer an inducible defect.

with aspirin, statins, and ACE inhibitors. Patients who demonstrated resolution of MPI abnormalities had the greatest increase in the use of these medications, whereas patients with new inducible ischemia had a much lower increase. The observed resolution of inducible ischemia was also a harbinger of the low cardiac event rates that subsequently emerged as a key finding of the DIAD study.

\section{CARDIAC OUTCOME AFTER 5 YEARS}

The predefined primary outcomes of the DIAD study were cardiac death and non-fatal myocardial infarction, the so-called hard events associated with cardiovascular disease in patients with diabetes. The outcome analysis showed the unanticipated and encouraging result, that the 5-year event rate was low: only $2.9 \%$ or an average of $0.6 \%$ per year. ${ }^{1}$ The cardiac deaths were few, but of concern, all except one, were sudden. Most of the myocardial infarctions were non-ST segment elevation infarcts and did not evolve to large Q-wave myocardial infarcts. DIAD subjects were also followed for the development of CHF, unstable angina, or coronary revascularization as secondary cardiac events. However, the secondary event rate was also quite low: $4.4 \%$ or an average of $0.9 \%$ per year.

The key outcome feature of the DIAD study was that screening had no effect on either primary (Figure 2) or secondary cardiac event rates. The favorable outcomes of the DIAD patient could not be explained by 


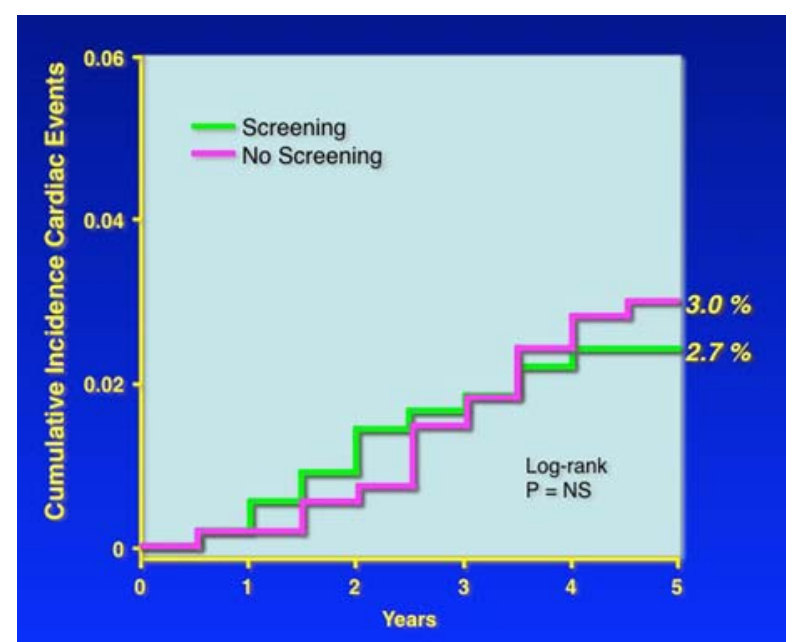

Figure 2. Cumulative incidence of cardiac events (cardiac death and non-fatal myocardial infarction) in 561 DIAD participants randomized to systematic baseline screening with adenosine Sestamibi SPECT imaging (green) and in 562 participants randomized to receive no screening (magenta). The outcome in both groups was not different after 5 years follow-up. (modified from Ref. 1).

revascularization, and in fact, the overall revascularization rate was low with $5.5 \%$ in the screening group and $7.8 \%$ in the no-screening group undergoing PTCA or CABG.

\section{TREATMENT AFTER RANDOMIZATION}

Screening per se does not improve cardiac outcomes unless coupled to additional therapies that actually are effective in preventing coronary events. One important aspect of the DIAD study is that participants were treated at the discretion of their own physicians. There was no protocol-mandated treatment of patients with abnormal screening results. Thus, the management of patients in the DIAD study reflects contemporary clinical practice in dealing with asymptomatic ischemia in diabetic patients. Screening did lead to a modest amount $(4.4 \%)$ of early ( $<120$ days) coronary angiography, whereas only $0.5 \%$ of subjects in the no screening group underwent early angiography. Of note, only 5 of $33(15 \%)$ patients with moderate or large defects were referred for coronary angiography by their health care providers. Ultimately, the rates of coronary angiography (14\% and $12 \%)$ and coronary revascularization $(5.5 \%$ and $7.8 \%$ ) were not different in screened and not screened participants. Although arguments could be made that this failed to provide potentially beneficial revascularization to those with significant inducible ischemia, one patient with a large MPI defect had sudden cardiac death 1 year after coronary bypass surgery.

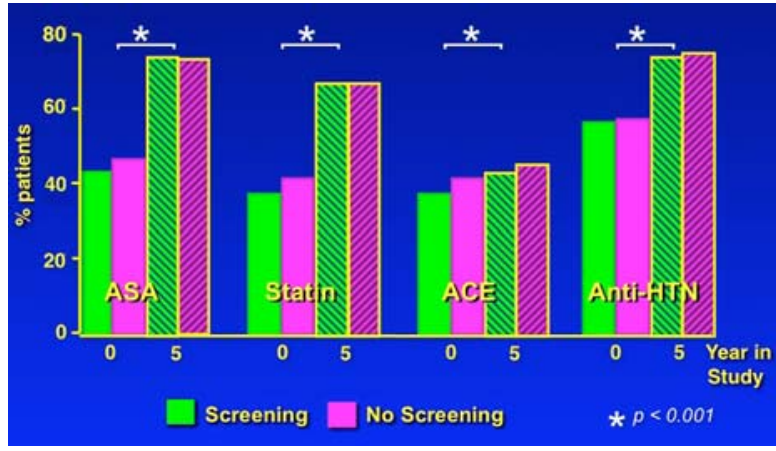

Figure 3. Use of primary medical prevention at baseline of the DIAD study and after 5 years follow-up. In both randomized groups (screening: green and no screening: magenta) there was a significant and equivalent increase in the use of aspirin (ASA), statins, angiotensin-converting enzyme (ACE) inhibitor, and antihypertensive medication from baseline (year 0 ) to end of follow-up (year 5).

Of the 28 participants with moderate or large defects who did not undergo coronary angiography, only 3 had cardiac events.

Another argument often proposed in support of screening is that the results will lead to the intensification of medications that have preventative benefit in diabetic patients. However, when changes in medical treatment were compared in the screening group and no screening group, a similar intensification of primary medical prevention was noted in the no screening group as was observed in the screening group (Figure 3).

Thus the DIAD study reveals a relatively low prevalence of silent myocardial ischemia at the outset of the study, resolution of a substantial portion of inducible ischemia after 3 years and overall a low cardiac event rate over 5 years. These findings all consistently support the favorable course of patients with asymptomatic diabetes. The favorable outcomes are likely ascribed in part to the aggressive recommendations for risk factor modification published by the American Diabetes Association in 2001 (shortly after the start of the DIAD study). The primary physicians of DIAD subjects took these recommendations to heart with significant intensification of medical management, likely affecting the overall outcome favorably. The favorable effects of optimal medical treatment in the DIAD study has now been recapitulated by the excellent results of the COURAGE, INSPIRE, and the BARI-2D trials. ${ }^{15-17}$

\section{PRACTICAL IMPLICATIONS}

Although screening had no impact on the outcomes in DIAD, it is noteworthy that stress MPI did effectively stratify patients into higher-risk (moderate-large defects and ischemic ECG) and low-risk (small defects or 


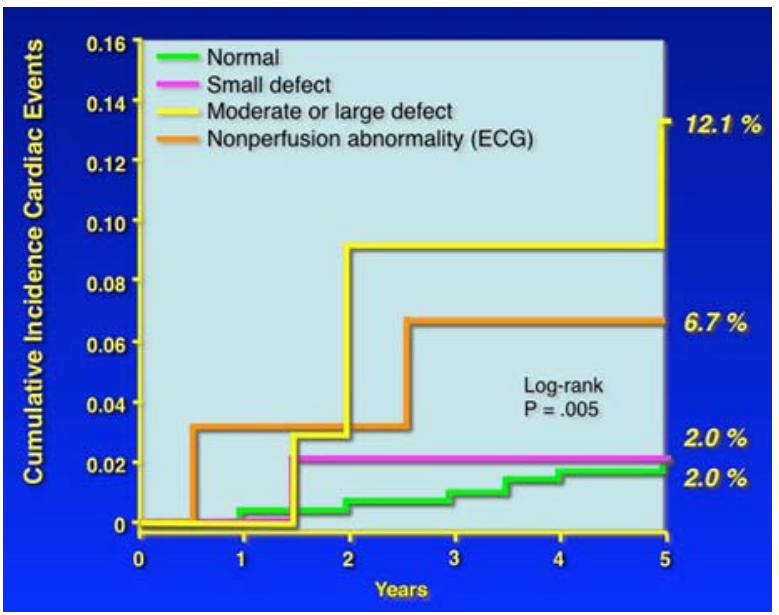

Figure 4. Cumulative incidence of cardiac events (cardiac death and non-fatal myocardial infarction) according to the results of systematic screening with adenosine Sestamibi SPECT imaging. Participants with moderate or large myocardial perfusion defects and with ischemic ECG changes during adenosine infusion had significantly worse outcome than participants with small or no defects.

normal MPI) subsets (Figure 4). However, because the overall outcomes in screened and unscreened patients were similar, DIAD indicates that blanket or routine screening of all patients with diabetes is not justified. Rather the generalized approach of selective clinically indicated diagnostic testing (stress tests or coronary angiography) appears to be preferable.

On the other hand, there were adverse cardiac outcomes in both screened patients and unscreened patients. Thus, the DIAD results do not exclude the possibility that strategies to better identify patients at higher risk coupled with more effective treatment strategies might prove effective for screening in the future.

\section{COST OF SCREENING}

In the current era of the health care financial crisis, the costs of all testing strategies are being carefully scrutinized. Routine screening of asymptomatic people with type 2 diabetes with specialized cardiac testing is likely to prove prohibitively expensive, irrespective of the approach. For instance, in the US alone there are an estimated 14 million patients with diabetes without known CAD. Routine screening with SPECT MPI (at Medicare reimbursement rate of $\$ 809$ per test) would cost a staggering $\$ 13.5$ billion.

It has been suggested that $\mathrm{CT}$ pre-screening for coronary artery calcium might be a more effective means for identifying patients with diabetes who then would be appropriate for additional cardiac testing. ${ }^{18}$ This approach too would engender substantial health care costs. Assuming the cost of calcium scoring at $\$ 300$, the cost of pre-screening would be $\$ 4.2$ billion. If $40 \%$ of diabetics have coronary artery calcification ${ }^{19}$, screening these 5.6 million patients with SPECT MPI would still cost $\$ 4.5$ billion, leading to a total screening cost of $\$ 8.7$ billion. This strategy also is difficult to justify when the overall annual cardiac event rate is $<1 \%$ and revascularization has not been proven to improve the outcomes of these higher-risk subjects.

\section{PREDICTORS OF CARDIAC EVENTS AND POSITIVE PREDICTIVE VALUE}

Better risk stratification based on clinical characteristics might be possible. Peripheral vascular disease, cardiac autonomic dysfunction, and diabetic peripheral neuropathy all appeared to be significant predictors of myocardial infarction or cardiac death in DIAD. However, their positive predictive values were low, i.e., $6.9 \%$ for cardiac autonomic dysfunction, $8.7 \%$ for peripheral vascular disease, and $2.7 \%$ for neuropathy. Further analysis of DIAD and other studies might provide additional insight into models that might in combination identify subjects at higher risk who might be appropriate for future research.

\section{CONCLUSION}

Thus, the DIAD study results indicate that routine screening of asymptomatic patients with diabetes is not justified, because of the relatively low yield of significant abnormalities, the low overall cardiac event rate with contemporary medical therapy, and the lack of impact of screening on events.

\section{References}

1. Young LH, Wackers FJ, Chyun DA, et al. Cardiac outcomes after screening for asymptomatic coronary artery disease in patients with type 2 diabetes: The DIAD study: A randomized controlled trial. J Am Med Assoc 2009;301:1547-55.

2. Wackers FJ, Young LH, Inzucchi SE, et al. Detection of silent myocardial ischemia in asymptomatic diabetic subjects: The DIAD study. Diabetes Care 2004;27:1954-61.

3. Wackers FJ, Chyun DA, Young LH, et al. Resolution of asymptomatic myocardial ischemia in patients with type 2 diabetes in the Detection of Ischemia in Asymptomatic Diabetics (DIAD) study. Diabetes Care 2007;30:892-8.

4. Janand-Delenne B, Savin B, Habib G, Bory M, Vague P, Lassmann-Vague V. Silent myocardial ischemia in patients with diabetes. Diabetes Care 1999;22:1396-400.

5. Kang X, Berman DS, Lewin HC, Cohen I, Friedman JD, Germano $\mathrm{G}$, et al. Incremental prognostic value of myocardial perfusion single photon emission computed tomography in patients with diabetes mellitus. Am Heart J 1999;138:1025-32. 
6. Penfornis A, Zimmermann C, Boumal D, et al. Use of dobutamine stress echocardiography in detecting silent myocardial ischaemia in asymptomatic diabetic patients: A comparison with thallium scintigraphy and exercise testing. Diabet Med 2001;18:900-5.

7. Gazzaruso C, Garzaniti A, Giordanetti S, Falcone C, De Amici E, Geroldi D, et al. Assessment of asymptomatic coronary artery disease in apparently uncomplicated type 2 diabetic patients. Diabetes Care 2002;25:1418-24.

8. DeLorenzo A, Lima RS, Siqueira-Filho AG, Pantoja MR. Prevalence and prognostic value of perfusion defects detected by stress technetium-99m sestamibi myocardial perfusion single-photon emission computed tomography in asymptomatic patients with diabetes mellitus and no known coronary artery disease. Am J Cardiol 2002;90:827-32.

9. Schinkel AF, Elhendy A, van Domburg RT, Bax JJ, Vourvouri EC, Sozzi FB, et al. Prognostic value of dobutamine-atropine stress myocardial perfusion imaging in patients with diabetes. Diabetes Care 2002;25:1637-43.

10. Giri S, Shaw LJ, Murthy DR, et al. Impact of diabetes on the risk stratification using stress single-photon emission computed tomography myocardial perfusion imaging in patients with symptoms. Circulation 2002;105:32-40.

11. Berman DS, Kang X, Hayes SW, Friedman JD, Cohen I, Abidov A, et al. Adenosine myocardial perfusion single-photon emission computed tomography in women compared with men. Impact of diabetes mellitus on incremental prognostic value and effect on patient management. J Am Coll Cardiol 2003;41:1125-33.

12. Prior JO, Monbaron D, Koehli M, Calcagni ML, Ruiz J, Bischof DA. Prevalence of symptomatic and silent stress-induced perfusion defects in diabetic patients with suspected coronary artery disease referred for myocardial perfusion scintigraphy. Eur J Nucl Med Mol Imaging 2005;32:60-9.

13. Rajagopalan N, Miller TD, Hodge DO, Frye RL, Gibbons RJ. Identifying high-risk asymptomatic diabetic patients who are candidates for screening stress single-photon emission computed tomography imaging. J Am Coll Cardiol 2005;45:43-9.

14. Milan Study on Atherosclerosis and Diabetes Group. Prevalence of unrecognized silent myocardial ischemia and its association with atherosclerotic factors in noninsulin-dependent diabetes mellitus. Am J Cardiol 1997;79:134-9.

15. Boden WE, O'Rourke RA, Teo KK, Hartigan PM, Maron DJ, Kostuk WJ, et al. Optimal medical therapy with or without PCI for stable coronary artery disease. N Eng J Med 2007;356:1503-16.

16. Mahmarian JJ, Dakik HA, Filipchuk NG, Shaw LJ, Iskander SS, Ruddy TD, et al. An initial strategy of intense medical therapy is comparable to that of coronary revascularization for suppression of scintigraphic ischemia in high-risk but stable survivors of acute myocardial infarction. J Am Coll Cardiol 2006;48:2458-67.

17. BARI The 2D Study Group. A randomized trial of therapies for type 2 diabetes and coronary artery disease. $\mathrm{N}$ Eng $\mathrm{J}$ Med 2009;360:2503-15.

18. Anand DV, Lim E, Hopkins D, et al. Risk stratification in uncomplicated type 2 diabetes: Prospective evaluation of the combined use of coronary artery calcium imaging and selective myocardial perfusion scintigraphy. Eur Heart J 2006;27:713-21.

19. Raggi P, Shaw LJ, Berman DS, Callister TQ. Prognostic value of coronary calcium screening in subjects with and without diabetes. J Am Coll Cardiol 2004;43:1663-9. 